\title{
Preclinical Evidence Supporting Early Initiation of Citalopram Treatment in Machado-Joseph Disease
}

\author{
Sofia Esteves ${ }^{1,2}$. Stéphanie Oliveira ${ }^{1,2}$. Sara Duarte-Silva ${ }^{1,2} \cdot$ Daniela Cunha-Garcia $^{1,2}$ - Andreia Teixeira-Castro ${ }^{1,2,3}$. \\ Patrícia Maciel ${ }^{1,2}$ (D)
}

Received: 8 June 2018 / Accepted: 23 August 2018

(C) Springer Science+Business Media, LLC, part of Springer Nature 2018

\begin{abstract}
Spinocerebellar ataxias are dominantly inherited neurodegenerative disorders with no disease-modifying treatment. We previously identified the selective serotonin reuptake inhibitor citalopram as a safe and effective drug to be repurposed for Machado-Joseph disease. Pre-symptomatic treatment of transgenic (CMVMJD135) mice strikingly ameliorated mutant ataxin-3 (ATXN3) pathogenesis. Here, we asked whether citalopram treatment initiated at a post-symptomatic age would still show efficacy. We used a cohort of CMVMJD135 mice that shows increased phenotypic severity and faster disease progression (CMVMJD135hi) compared to the mice used in the first trial. Groups of hemizygous CMVMJD135hi mice were orally treated with citalopram. Behavior, protein analysis, and pathology assessment were performed blindly to treatment. Our results show that even when initiated after symptom onset, treatment of CMVMJD135hi mice with citalopram ameliorated motor coordination and balance, attenuating disease progression, albeit to a lesser extent than that seen with pre-symptomatic treatment initiation. There was no impact on ATXN3 aggregation, which contrasts with the robust reduction in ATXN3-positive inclusions observed in CMVMJD135 mice, when treated pre-symptomatically. Postsymptomatic treatment of CMVMJD135hi mice revealed, however, a limited neuroprotective effect by showing a tendency to repair cerebellar calbindin staining, and to increase the number of motor neurons and of NeuN-positive cells in certain brain regions. While supporting that early initiation of treatment with citalopram leads to a marked increase in efficacy, these results strengthen our previous observation that modulation of serotonergic signaling by citalopram is a promising therapeutic approach for Machado-Joseph disease even after symptom onset.
\end{abstract}

Keywords Spinocerebellar ataxia type 3 - Selective serotonin reuptake inhibitor - Citalopram - Post-symptomatic treatment . Transgenic model

Sofia Esteves and Stéphanie Oliveira contributed equaly to the work.

Andreia Teixeira-Castro and Patrícia Maciel share co-senior authorship.

Electronic supplementary material The online version of this article (https://doi.org/10.1007/s12035-018-1332-1) contains supplementary material, which is available to authorized users.

Andreia Teixeira-Castro

accastro@med.uminho.pt

$\triangle$ Patrícia Maciel

pmaciel@med.uminho.pt

1 Life and Health Sciences Research Institute (ICVS), School of Medicine, University of Minho, Campus of Gualtar, 4710-057 Braga, Portugal

2 ICVS/3B's - PT Government Associate Laboratory, Braga/ Guimarães, Portugal

3 Department of Molecular Biosciences, Northwestern University, Evanston, IL 60208, USA

\author{
Abbreviations \\ 5-HT Serotonin \\ MJD Machado-Joseph disease \\ SSRI Selective serotonin reuptake inhibitor.
}

\section{Introduction}

Machado-Joseph disease (MJD) or spinocerebellar ataxia type 3 is a clinically heterogeneous neurodegenerative disease $[1,2]$, caused by an expansion of a cytosine-adenineguanine (CAG) repeat tract in the ATXN3 gene, which encodes for an abnormally long polyglutamine (polyQ) segment in the ataxin-3 protein (ATXN3) [3]. The symptoms of MJD reflect the involvement of multiple neurological systems, which include ataxia, diplopia, dysphagia, 
dysarthria, and ophthalmoplegia, as well as fasciculations, amyotrophy, dystonia, and/or spasticity $[4,5]$. This disorder is characterized by a widespread neuronal degeneration affecting several regions of the central nervous system, such as the cerebellum, brainstem, basal ganglia, and spinal cord [6]. No effective disease-modifying treatment is currently available for MJD.

Citalopram is a safe, well-tolerated antidepressant that belongs to the group of the selective serotonin reuptake inhibitors (SSRIs) [7, 8]. These compounds share the same cellular target, the serotonin (5-HT) transporter (SERT), which is responsible for 5-HT uptake into serotonergic neurons [9]. While the exact mechanism of action of SSRIs has yet to be elucidated, these compounds may exert their antidepressant activity by inhibiting SERT and increasing extracellular levels of 5-HT [10].

Antidepressants are often prescribed to patients with neurodegenerative diseases to treat depression and anxiety symptoms [11]. Interestingly, several antidepressants have also shown neuroprotective effects in vitro, as well as in animal models of Alzheimer's disease, Parkinson's disease, Huntington's disease, or spinal and bulbar muscular atrophy [12-19].

We previously described an in vivo repurposing screen of 1200 FDA-approved small molecules and identified chemical suppressors of mutant ATXN3-induced neurotoxicity in Caenorhabditis elegans [20]. Through this unbiased approach, we found that citalopram and other SSRIs reduced mutant ATXN3 aggregation in C. elegans and restored its motor capacity. In this simple animal model, early initiation and prolonged treatment with citalopram resulted in increased therapeutic efficacy. Chronic citalopram treatment of CMVMJD135 mice [22], a vertebrate model of MJD, when initiated at a pre-symptomatic age, strikingly ameliorated their motor coordination impairments, reducing mutant ATXN3 aggregation and neuronal loss [20]. These results suggest that small-molecule modulation of serotonergic signaling in presymptomatic stages may represent a promising therapeutic approach for MJD.

To mimic the most frequent clinical situation of symptomdriven diagnosis and treatment of MJD patients, here, we asked whether citalopram treatment would still be effective to ameliorate mutant ATXN3-mediated pathogenesis if initiated after motor symptom installation in MJD mice.

Our results showed that, indeed, post-symptomatic citalopram treatment ameliorated loss of balance and motor coordination, attenuating disease progression in a cohort of MJD mice of increased disease severity (CMVMJD135hi). These results suggest that 5-HT recapture inhibition can be used as a disease-modifying therapy for MJD and perhaps other conformational disorders, even when initiated after symptom onset, but also support increased efficacy of early treatment initiation.

\section{Materials and Methods}

\section{Ethics Statement}

All animal procedures were conducted in accordance with European regulations (European Union Directive 2010/63/ EU). Animal facilities and the persons directly involved in animal experimentation (SE, SD-S, SO, AT-C) were certified by the Portuguese regulatory entity-Direcção Geral de Alimentação e Veterinária. All the protocols were approved by the Animal Ethics Committee of the Life and Health Sciences Research Institute, University of Minho. All experiments were designed with commitment to the principles of refinement, reduction, and replacement and performed according to FELASA guidelines to minimize discomfort, stress, and pain to the animals, with defined humane endpoints (20\% reduction of the body weight, inability to reach food and water, presence of wounds in the body, and dehydration) [23]. The status of specified pathogens of sentinel animals, maintained in the same animal room, was monitored throughout the study.

\section{Transgenic Mouse Model and Drug Administration}

CMVMJD135hi mice (background C57BL/6) were generated as previously described [22], but by selecting progenitor animals with a mean CAG repeat size of $138 \pm 5[ \pm$ standard deviation (SD)]. Animals were housed in a conventional animal facility at weaning, in groups of five animals, in filtertopped polysulfone cages $267 \times 207 \times 140 \mathrm{~mm}\left(370 \mathrm{~cm}^{2}\right.$ floor area) (Tecniplast, Buguggiate, Italy), using corncob bedding (Scobis Due, Mucedola SRL, Settimo Milanese, Italy). All animals were maintained under standard laboratory conditions: an artificial 12-h light/dark cycle (lights on from 8 am to $8 \mathrm{pm}$ ), with $21 \pm 1{ }^{\circ} \mathrm{C}$ of room temperature and a relative humidity of $50-60 \%$. Mice were fed with a standard diet (4RF25 during the gestation and postnatal periods, and 4RF21 after weaning; Mucedola SRL, Settimo Milanese, Italy) and water ad libitum. DNA extraction, animal genotyping, and CAG repeat size analyses were performed as previously described [24]. The mean CAG repeat size $[ \pm$ SD] for all transgenic mice used in this study, the CMVMJD135hi animals, was of $138 \pm 5$, distributed evenly among treatments (CMVMJD135hi $138 \pm 6$ and CMVMJD135hi cit $137 \pm 5$ ). Age-matched WT littermate animals were used as controls. Male mice were used in this study. The first symptom that appears in CMVMJD135 mice is loss of motor strength in the hanging wire test using an inverted grid, which is already installed at 6 weeks of age, followed by the appearance of motor uncoordination deficits [22]. To verify installation of motor symptoms in CMVMJD135hi mice, we evaluated balance and motor coordination of the mice on the balance beam walk and motor swimming paradigms [25]. Transgenic 
animals showed increased latency time to reach the safe platforms in both behavioral tests when compared to WT animals at 10 weeks of age. Therefore, treatment was initiated at 11 weeks of age. We administrated citalopram hydrobromide (CAS 59729-32-7, kindly provided by H. Lundbeck A/S, Denmark) in the drinking water at a dosage of $8 \mathrm{mg} / \mathrm{kg}$. Citalopram-supplemented water was changed three times per week throughout the entire duration of the trial to ensure drug activity. The trial was terminated at 28 weeks of age, when most of the vehicle-treated transgenic animals could not complete any of the motor tests (e.g., balance beam walk test, motor swimming test, and footprints).

\section{Experimental Design}

ARRIVE guidelines were followed throughout the study [26]. Experimental design was based on power analyses for optimization of sample size, as described previously [20]. Mouse sample size calculations were previously performed for each behavioral test and pathological analyses, assuming a power of 0.95 and 0.8 , respectively, and a significance level of $p<0.05$ [20]. Transgenic and non-transgenic drug- and placebo-treated animals were alternately housed and assigned to treatment. All behavioral experiments and neuropathological analyses were conducted by researchers who were blind to genotype and treatment. We used groups of 13-16 animals per genotype/treatment for behavioral tests and groups of four to six animals for quantification of ATXN3 intranuclear inclusions, assessment of astrogliosis, cell counts, and Western blot analyses. Regarding genotype-phenotype correlations, data obtained in the previous preclinical trial using vehicletreated CMVMJD135 mice [20] was used for correlation analyses in conjugation with data from the CMVMJD135hi mice (present study). Motor performance in the balance beam walk and motor swimming tests were analyzed at 26 weeks of age for all animals $(n=27)$ and correlated to their CAG repeat number.

\section{Behavioral Assessment}

Behavioral assessment was performed during the diurnal period, with five males per cage, including CMVMJD135hi hemizygous transgenic mice and WT littermates $(n=15-16$ per genotype) treated with citalopram or with vehicle (water). Body weight was registered throughout the study. All behavioral tests started in a symptomatic stage ( 10 weeks) and were conducted until 26 or 28 weeks of age. Neurological testing included (1) a selection of tests from the SHIRPA protocol, namely assessment of tremors, gait quality, and limb clasping $[27,28]$; (2) footprinting analysis and stride length quantification; (3) balance beam walk (12-mm and $27-\mathrm{mm}$ square beams); and (4) motor swimming tests. All behavioral tests were performed as previously described [20, 22, 24]. Briefly, tremors assessment was performed while the animals were in a viewing jar and scored (as absent, mild (discontinuous tremor), or severe (continuous tremor)) while animals were immobile. Gait quality was assessed by the experimenter in an open arena $(55 \times 33 \times 18 \mathrm{~cm})$. Freely moving animals were scored as normal, abnormal (incorrect posture of the body and tail, with decreased distance over the ground), and limited (very limited movement). To determine limb clasping, animals were picked up by the tail and slowly descending towards a horizontal surface. Mice were observed and scored as absent (extension of the hindlimbs), mild (contraction observed in one of the limbs), or severe (contraction observed in both limbs). To register footprint patterns, the fore and hind paws of the animals were coated with non-toxic red and black inks, respectively. Animals were allowed to walk along a $100 \mathrm{~cm}$ long $\times$ $4.2 \mathrm{~cm}$ width $\times 10 \mathrm{~cm}$ height inclined runway (a clean paper sheet was used for each mouse) in the direction of an enclosed safe black box. Stride length was obtained by measuring the distance between two paw prints. Three values were obtained in six consecutive steps and the mean of the three values was used. The same consecutive steps were used to evaluate the severity of foot dragging. Mice were scored as absent/mild $=$ 0 (up to three steps), mild $=1$ (more than three steps out of six), and severe $=2$ (all steps out of six). Balance beam walk test comprised 3 days of training (three trials per animal) in the $12-\mathrm{mm}$ square beam, and, in the fourth day, animals were tested in the $12-\mathrm{mm}$ and/or in the $27-\mathrm{mm}$ square beam (two trials per animal were recorded). If the animal turned around or fell from the beam, the trial was considered invalid. Each animal is allowed to fail twice. The time the animals took to cross the beams was recorded, and if animals stopped, this time was discounted. The percentage of therapeutic efficacy was calculated taking the mean latency time to reach the safe platform in the beam walk test of transgenic mice and subtracting the mean latency time of WT mice in each preclinical trial, to obtain a reference value. This value corresponded to $100 \%$ of therapeutic efficacy in that trial (i.e., assuming that the best possible drug will make the transgenic animals behave like WT). The difference between latency time of vehicle-treated transgenic mice and latency time of cit-treated mouse of the same trial, relative to the value of $100 \%$ efficacy, corresponded to the percentage of therapeutic effect. This was calculated at 26 weeks of age both in the pre[20] and post-symptomatic trials (this study). In the motor swimming test, mice were trained for two consecutive days (animals were allowed three trials) to cross a transparent acrylic water tank (100 cm long) to a safe (black acrylic-made) platform at the end. The latency to cross this tank was registered from a $60-\mathrm{cm}$ distance (the initiation position was marked with a blue line). The water temperature was maintained at $23{ }^{\circ} \mathrm{C}$ using a thermostat. Animals were tested for three consecutive days (two trials per animal) and the latency to cross the tank assessed by the experimenter. 


\section{Immunohistochemistry and Quantification of Neuronal Inclusions}

Twenty-eight-week-old WT and CMVMJD135hi littermate mice, vehicle- and citalopram-treated ( $n=4-6$ per group), were deeply anesthetized [a mixture of ketamine hydrochloride $(150 \mathrm{mg} / \mathrm{kg})$ plus medetomidine $(0.3 \mathrm{mg} / \mathrm{kg})]$ and transcardially perfused with phosphate-buffered saline (PBS) followed by $4 \%$ paraformaldehyde (PFA) (Panreac, USA). Brains were removed and postfixed overnight in PFA and either embedded in paraffin or transferred to a $30 \%$ sucrose solution for vibratome processing. Spinal cord tissues were postfixed overnight in PFA and transferred to a $30 \%$ sucrose solution for vibratome processing. Brain slices $(4-\mu \mathrm{m}$-thick paraffin sections or $40-\mu \mathrm{m}$-thick vibratome sections) and spinal cord vibratome sections ( $50-\mu \mathrm{m}$-thick) were subjected to antigen retrieval and then incubated with mouse anti-ATXN3 (1H9) (1:750 for paraffin and 1:500 for vibratome sections, MAB5360, Millipore), rabbit anti-GFAP (1:500, Dako Corporation), goat anti-choline acetyltransferase (ChAT, 1:500, AB144P, Millipore), mouse anti-neuronal nuclei (NeuN, 1:100, MAB377, Millipore), and rabbit anticalbindin D-28K (1:1000, AB1778, Millipore) antibodies, which were detected by incubation with a biotinylated antipolyvalent antibody, followed by detection through biotinstreptavidin coupled to horseradish peroxidase and reaction with the DAB (3,3'-diaminobenzidine) substrate (Lab Vision $^{\mathrm{TM}}$ Ultra-Vision ${ }^{\mathrm{TM}}$ Detection kit, Thermo Scientific or VECTASTAIN ${ }^{\circledR}$ Elite ${ }^{\circledR}$ ABC-HRP Kit, Vector Laboratories). Brain and spinal cord sections were counterstained with hematoxylin $25 \%$ following standard procedures. Quantification of ATXN3-positive inclusions was performed in the pontine nuclei $(\mathrm{PN})$, reticulotegmental nucleus of the pons (RtTg), facial motor nuclei $(7 \mathrm{~N})$, lateral reticular nuclei (LRt), vestibular nuclei (VN), deep cerebellar nuclei (DCN), and lumbar spinal cord (LSC). Number of GFAP-positive cells was determined in the substantia nigra (SN) and of calbindin-positive cells in the Purkinje cells of the cerebellar cortex (PJc CBX). Counts of ChAT-positive cells were conducted in the LSC, 7N, and dorsal striatum (DS). Quantification of NeuN-positive neurons was performed in the PN, DCN, and VN. All quantifications were carried out in either vehicle- or citalopram-treated animals ( $n=4$ for each condition, 4 slices per animal) and normalized to total area and to WT- or transgenic-vehicle controls using an Olympus BX51 stereological microscope (Olympus, Japan) and the Visiopharm integrator system software (Visopharm, Denmark) as previously described [24].

\section{Immunoblotting Analysis}

Protein isolation from mouse brainstem, cerebellum, and spinal cord tissue and Western blotting were performed as previously described [24]. Briefly, the blots were incubated overnight at $4{ }^{\circ} \mathrm{C}$ with the primary antibodies mouse antiATXN3 (1H9) (1:2000, MAB5360, Millipore) and mouse anti-tubulin (1:5000, T5168, Sigma). Mouse ATXN3 and tubulin were used as loading controls. Antibody affinity was detected by chemiluminescence, and Western blot quantifications were performed using Chemidoc XRS Software with ImageLab Software (Bio-Rad), following the manufacturer's instructions.

\section{Statistical Analysis}

Normality of variance assumptions was evaluated with Kolmogorov-Smirnov (K-S) or Shapiro-Wilk (S-W) test (chosen according to $n$ value of each experiment), absolute value of skewness, kurtosis, and $Z$-score. Substantial departure from normality was considered when K-S or S-W test showed $P<0.05$, absolute value of skewness $>2$, absolute value of kurtosis $>7$ and $Z$-score $>1.9$ [29]. Continuous variables with normal distributions and with homogeneity of variance (evaluated by Levene's test) were analyzed with repeated-measures two-way ANOVA for longitudinal multiple comparisons, using genotype and treatment as factors. One-way ANOVA was used for paired comparisons, using Tukey test for post hoc comparisons. Variables lacking homogeneity of variances were analyzed with parametric tests using Welch's correction (and Games-Howell was used for post hoc comparisons). Other variables were analyzed through non-parametric Mann-Whitney $U$ test or Kruskal-Wallis $H$ test for two or more groups, respectively. Genotype-phenotype correlations were analyzed using Pearson correlation coefficient (GraphPad). All statistical analyses were performed using SPSS 22.0 (SPSS Inc., Chicago, IL) and G-Power 3.1.9.2 (University Kiel, Germany). A critical value for significance of $P<0.05$ was used throughout the study. Crosses $(\times)$ represent CMVMJD135 and CMVMJD135hi comparisons, asterisks (*) represent WT and CMVMJD135hi comparisons, ampersands $(\&)$ represent WT versus WT cit comparisons, and number signs (\#) represent CMVMJD135hi and CMVMJD135hi cit comparisons.

\section{Results}

\section{Establishment of a Cohort of CMVMJD135 Mice with a More Severe Phenotype}

To determine the efficacy of post-symptomatic citalopram treatment in an animal model of disease with increased pathogenicity, we used a transgenic mouse model of MJD, the CMVMJD135, replicating patients' symptomatology by displaying progressive motor dysfunction and selective neuropathology [22]. As seen in MJD patients [30], the mean CAG 
repeat number in the CMVMJD135 mice correlates inversely with the age at onset of symptoms and directly with symptom severity and disease progression. This feature can be used to generate subsets of animals with distinct degrees of disease severity and progression rates. In this study, we chose to increase the mean CAG repeat length of the original CMVMJD135 mice, used in our previous study with citalopram [20], by 10 repeats (CMVMJD135hi). The increase in mean CAG repeat size from 128 in CMVMJD135 mice (CMVMJD135 vehicle group of our previous study) to 138 in CMVMJD135hi mice caused a significant aggravation of the motor uncoordination and loss of balance, as measured by the motor swimming and balance beam walk behavior paradigms (Fig. 1b, c). CMVMJD135hi mice also showed an accelerated rate of disease progression (ANOVA repeated measures, $P=0.042$ for motor swimming and $P<0.0001$ for balance beam tests). Latency time in both behavioral tasks significantly correlated with the CAG repeat length of the two groups (Pearson correlation coefficient $r^{2}=0.4037(n=27)$ and $r^{2}=0.6315$ $(n=22)$, respectively; $P<0.001)$ at 26 weeks of age (Fig. 1d, e). Age at onset of symptoms was significantly anticipated in CMVMJD135hi mice, by four or more weeks to a maximum of 16 weeks for stride length evaluation (Supplementary Table 1).

\section{Symptomatic Citalopram Treatment of CMVMJD135hi Mice Ameliorated Gait Quality}

At 10 weeks of age, CMVMJD135hi mice showed significant impairments in motor coordination and balance compared to WT animals (Supplementary Fig. 1). Oral chronic administration of citalopram ( $8 \mathrm{mg} / \mathrm{kg}$ ) or drinking water to CMVMJD135hi mice and to WT non-transgenic littermate controls was initiated at 11 weeks of age (study design shown in Fig. 2a). Assessment of mouse body weight throughout the duration of the trial (until 28 weeks of age; Fig. 2b) revealed no changes caused by citalopram treatment. Whereas some a
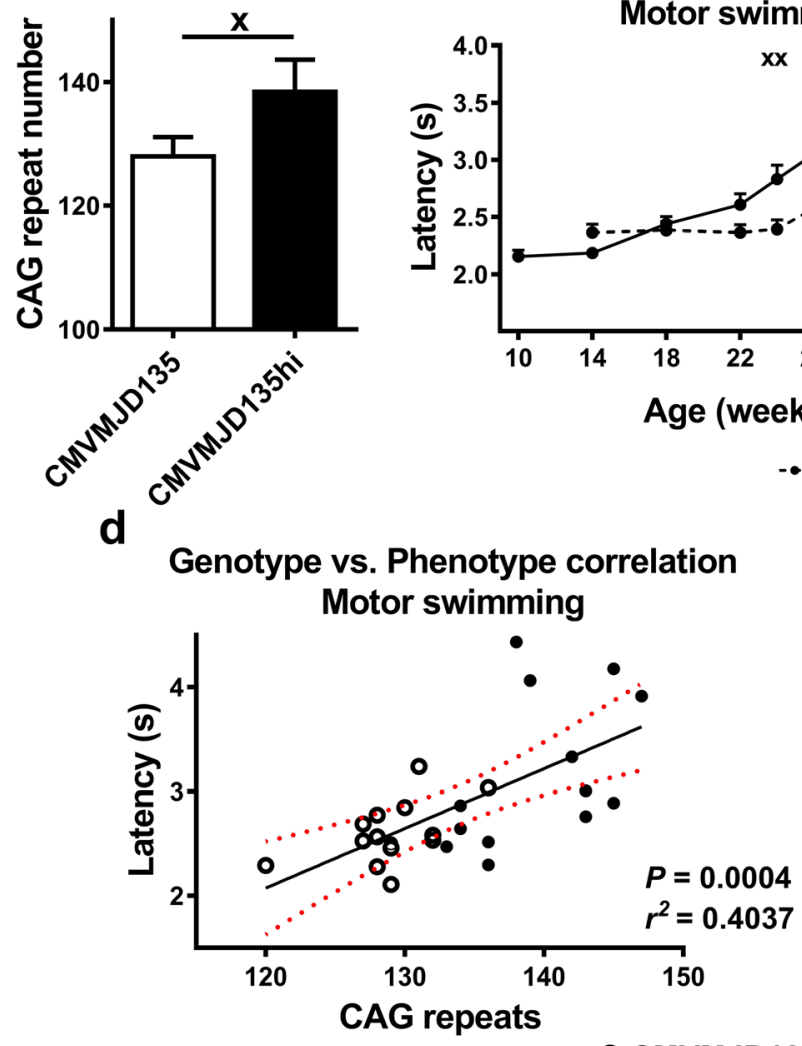

O CMVMJD135

Fig. 1 CMVMJD135hi mice present increased phenotype severity related to higher $(\mathrm{CAG}) \mathrm{n}$ repeat length. a CAG repeat number comparison between two independent mouse groups, the one used previously in the pre-symptomatic citalopram preclinical trial (CMVMJD135) and CMVMJD135hi mice (present study) $(n=27)$. Significant differences were observed between CMVMJD135 and CMVMJD135hi mouse groups (treated with vehicle) in preclinical trials regarding their performance in the $\mathbf{b}$ motor swimming test and in the $\mathbf{c}$ balance beam walk test (12-mm square). Genotype versus phenotype
C

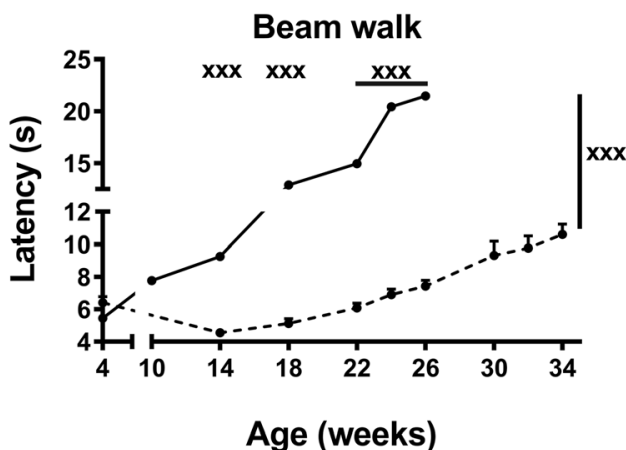

$\rightarrow$ CMVMJD135hi

e

Genotype vs. Phenotype correlation Beam walk

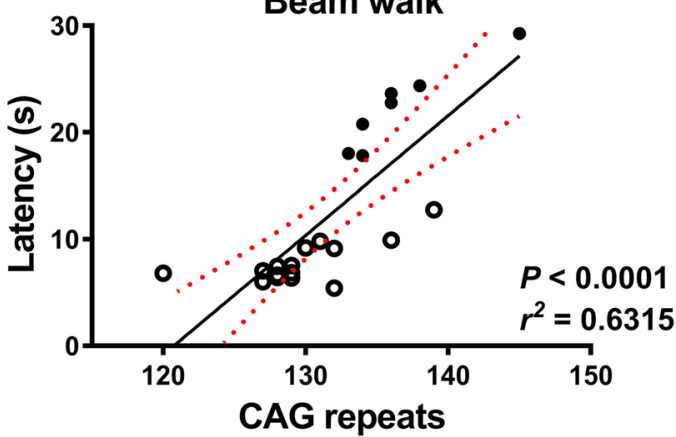

CMVMJD135hi

correlation for the d motor swimming test (CMVMJD135 + CMVMJD135hi, $n=27$ ) and e $12-\mathrm{mm}$ square balance beam walk (CMVMJD135 + CMVMJD135hi, $n=22$ ) at 26 weeks of age. Open circles refer to CMVMJD135 group and closed circles refer to CMVMJD135hi group. Data are presented as mean \pm SD (a) or mean \pm $\operatorname{SEM}(\mathbf{b}, \mathbf{c}),{ }^{\mathrm{x}} P<0.05,{ }^{\mathrm{xx}} P<0.01$, and ${ }^{\mathrm{xxx}} P<0.001$ (Student's $t$ test, repeated-measures two-way ANOVA, Tukey correction, and Pearson correlation coefficient). $r^{2}$ square of the Pearson correlation coefficient 
a

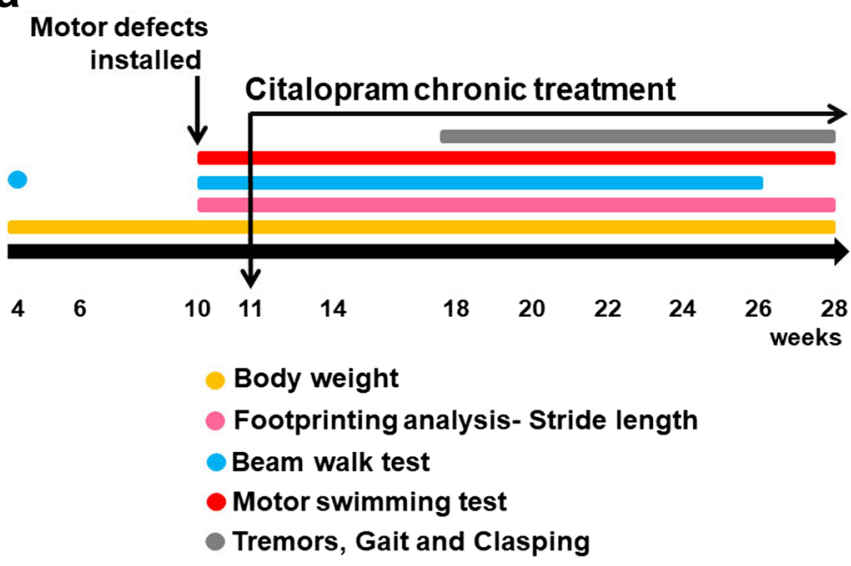

C

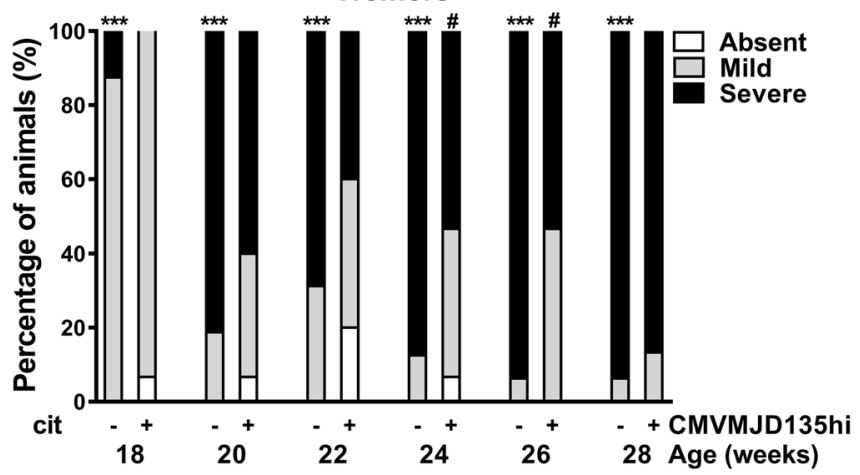

e

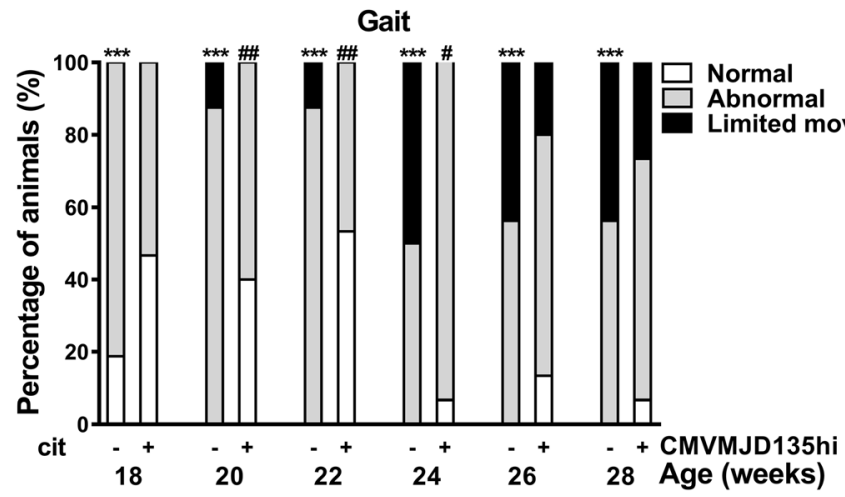

Fig. 2 Chronic post-symptomatic citalopram treatment ameliorated gait quality of CMVMJD135hi mice. a Schematic representation of the preclinical therapeutic trial design. b Body weight, $\mathbf{c}$ tremors, $\mathbf{d}$ limb clasping, e gait quality, and f footprint stride length were evaluated until 28 weeks of age in WT and CMVMJD135hi vehicle- and citalopramtreated mice (WT, $n=13$; WT cit, $n=17$; CMVMJD135hi, $n=16$; CMVMJD135hi cit, $n=15$ ). Mild improvements were observed for tremors, clasping, gait quality, and stride length, while no effect was observed in body weight upon treatment. Data are presented as mean \pm SEM; $* P<0.05, * * P<0.01$, and $* * * P<0.001$. Asterisks $(*)$ indicate

neurological symptoms of CMVMJD135hi mice, like body tremors, showed only marginal improvements upon citalopram treatment (Fig. 2c), limb clasping and gait quality scores were restored until 26 weeks of age (Fig. 2d, e). In agreement with what was observed when citalopram was administrated before b
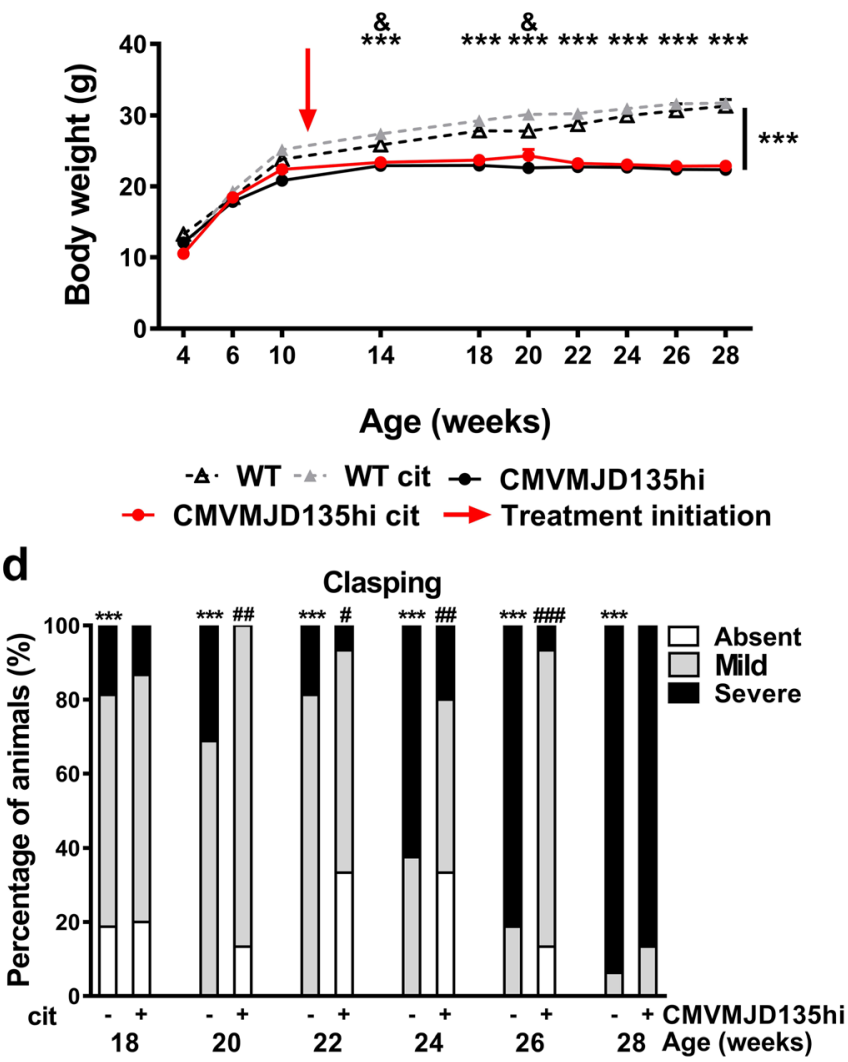

f

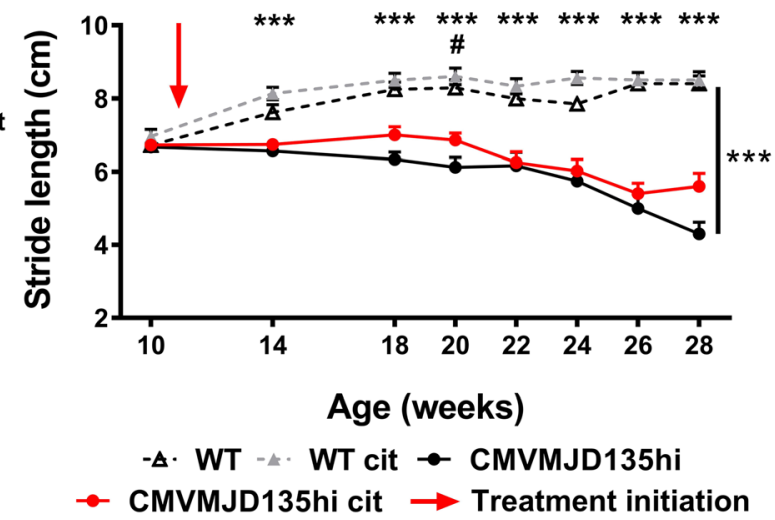

statistical significance between WT and CMVMJD135hi mice; the ampersands and number signs indicate statistical significance between WT and WT cit and between CMVMJD135hi and CMVMJD135hi cit mice, respectively. WT mice show no signs of tremors, altered limb clasping, or gait quality and are not represented in $\mathbf{c}, \mathbf{d}$, and e for simplicity (repeated-measures two-way ANOVA, one-way ANOVA, Tukey correction, and Mann Whitney $U$ test for non-continuous variables). The red arrow indicates age of treatment initiation at 11 weeks. cit citalopram

the onset of motor symptoms, treated CMVMJD135hi mice still displayed dragging of the paws (Supplementary Fig. 2); however, the stride length was significantly ameliorated at 20 weeks of age (Fig. 2f) in this trial, even though no changes were observed beyond this time point. 


\section{Post-symptomatic Treatment with Citalopram Improves Motor Coordination and Balance in CMVMJD135hi Mice}

Vehicle-treated CMVMJD135hi mice showed severe motor coordination and balance defects, which precluded testing of citalopram's beneficial effects at advanced stages of disease progression, as by 26 weeks, $40 \%$ of the animals were unable to cross the balance beam. Nevertheless, upon citalopram treatment, we observed a significant amelioration in motor performance in the different beam sizes analyzed, until 26 weeks of age (Fig. 3a, b). The animals that did not perform the test (40\%) at 26 weeks had a mean CAG repeat size of 140 $\pm 6[ \pm$ standard deviation (SD)] whereas the animals that were able to cross the beam (60\%) had a mean CAG repeat size of $136 \pm 4$, being this difference statistically significant (Student's $t$ test, $P=0.04$ ). However, among the mice that completed the task, citalopram-treated mice with a higher CAG repeat length did not take more time to traverse the beam, which suggests a disease-modifying effect for citalopram, being the slope of the correlation curve between CAG repeat length and latency to fall lower (Supplementary Fig. 3). In the motor swimming test, citalopram-treated CMVMJD135hi mice took less time to reach the safe platform (Fig. 3c), although not reaching statistical significance.

Overall, these results demonstrate that post-symptomatic citalopram treatment can still reduce the impairment in motor coordination and delay disease progression of MJD transgenic mice, although to a lesser extent than when the treatment is initiated before the onset of motor symptoms.

\section{Post-symptomatic Treatment with Citalopram Confers a Limited Neuroprotective Effect to CMVMJD135hi Mice}

The analysis of brain tissue of post-symptomatic citalopramtreated CMVMJD135hi mice suggested that the positive impact on animals' behavior may be accounted by the limited

Fig. 3 Citalopram post-symptomatic treatment improves balance and coordination of CMVMJD135hi mice. a Significant differences were observed in the 12-mm square balance beam walk (curve comparison over time $P<0.001,14-26$ weeks) and in the b $27-\mathrm{mm}$ square beam $(P<0.01,26$ weeks). At 26 weeks, data is shown for only $60 \%$ of the CMVMJD135hi vehicle-treated animals, since the other $40 \%$ were unable to complete this test. c In the motor swimming test, transgenic treated mice (CMVMJD135hi cit, $n=15$ ) took slightly less time to cross the swimming pool than vehicle-treated mice (CMVMJD135hi, $n=16$ ) (curve comparison over time $P>0.05,10-28$ weeks). Data are presented as mean $\pm \mathrm{SEM}, * P<0.05, * * P<0.01$, and $* * * P<0.001$. The asterisks (*) indicate statistical significance between WT and CMVMJD135hi mice; the number signs indicate statistical significance between CMVMJD135hi and CMVMJD135hi cit mice (repeated-measures twoway ANOVA and one-way ANOVA, Tukey correction). The red arrow indicates age of treatment initiation at 11 weeks neuroprotective effects exerted by the SSRI, even when administrated after symptom installation. Concerning mutant protein aggregation, and in contrast with pre-symptomatic treatment, the number of ATXN3 intranuclear inclusions in several areas of the brainstem and in the ventral horn of the lumbar spinal cord did not change when treatment was initiated at symptomatic ages (Fig. 4a and Supplementary Fig. 4a).

a
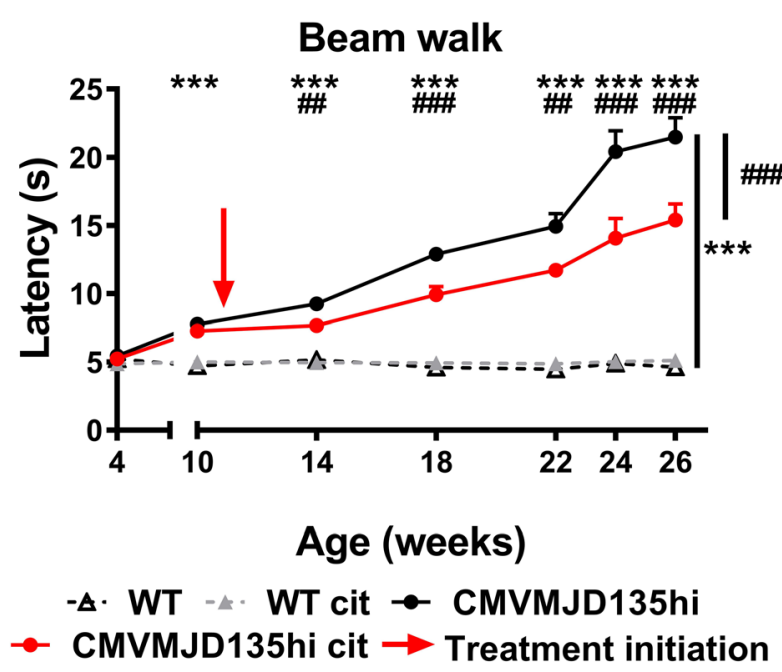

b

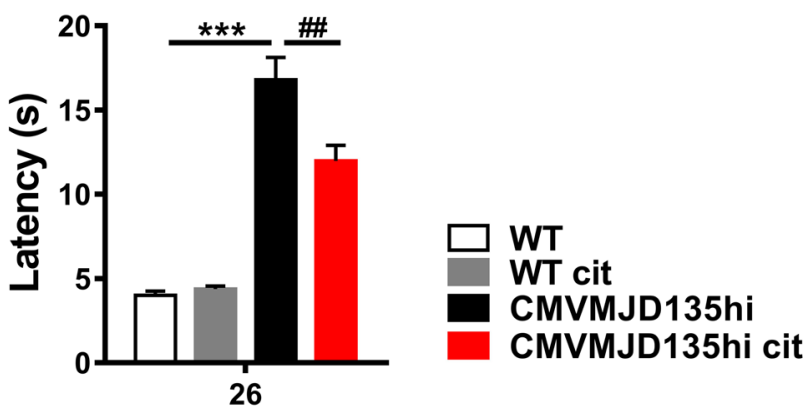

Age (weeks)

C
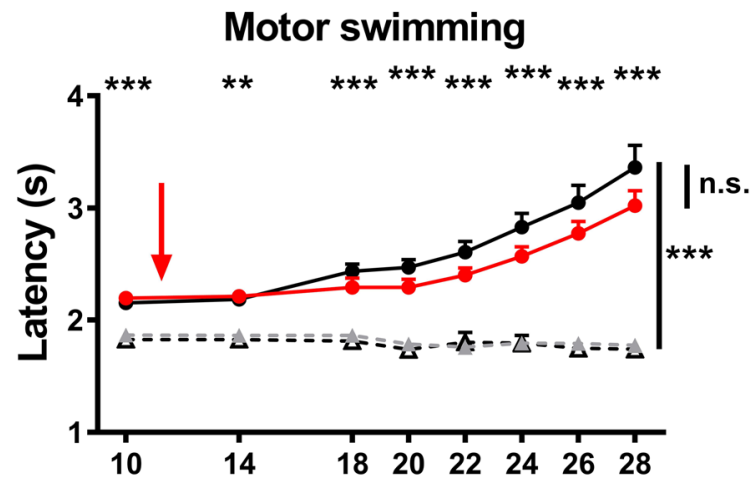

Age (weeks)

$-\Delta \cdot$ WT - WT cit $\rightarrow$ CMVMJD135hi

$\rightarrow$ CMVMJD135hi cit $\rightarrow$ Treatment initiation 

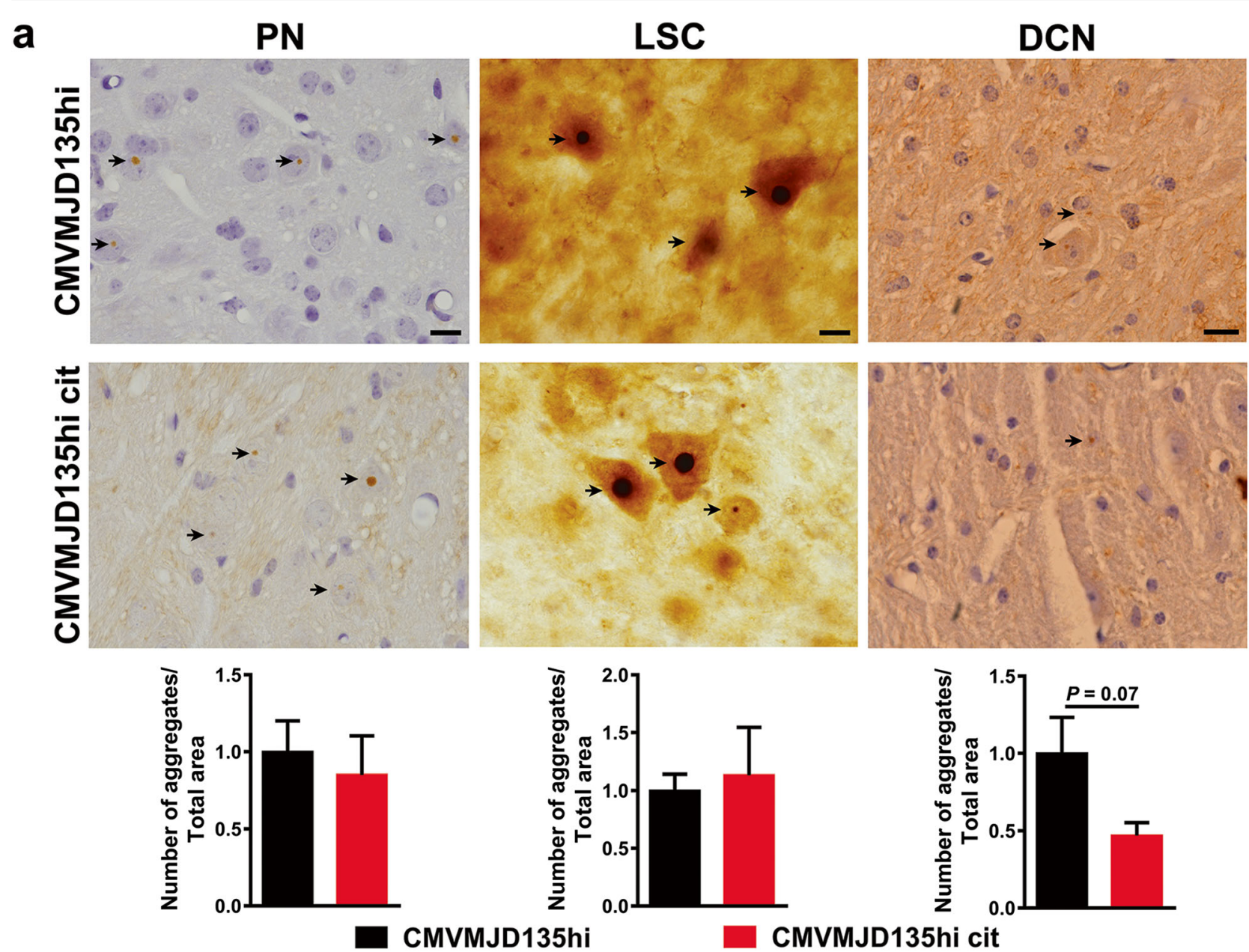

b

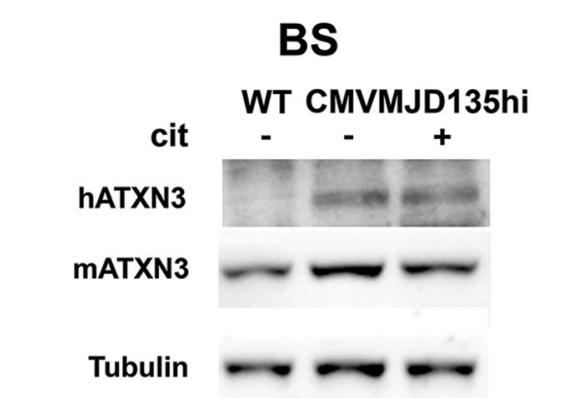

\section{CMVMJD135hi}
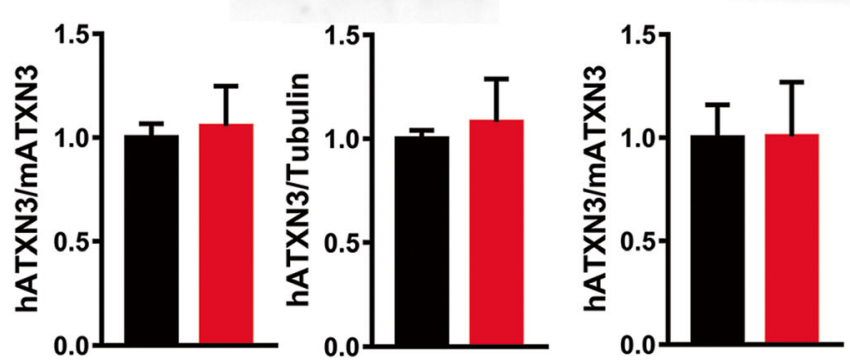

Fig. 4 Impact of post-symptomatic citalopram treatment of CMVMJD135hi mice on brain ATXN3 intranuclear inclusions and protein levels. a ATXN3-positive inclusions in the pontine nuclei (PN), lumbar spinal cord (LSC), and deep cerebellar nuclei (DCN) of vehicleand citalopram-treated CMVMJD135hi mice $(n=4,28$ weeks). b Brainstem (BS), spinal cord (SC), and cerebellum (CB) immunoblots and quantification of total human ATXN3 protein from vehicle- and
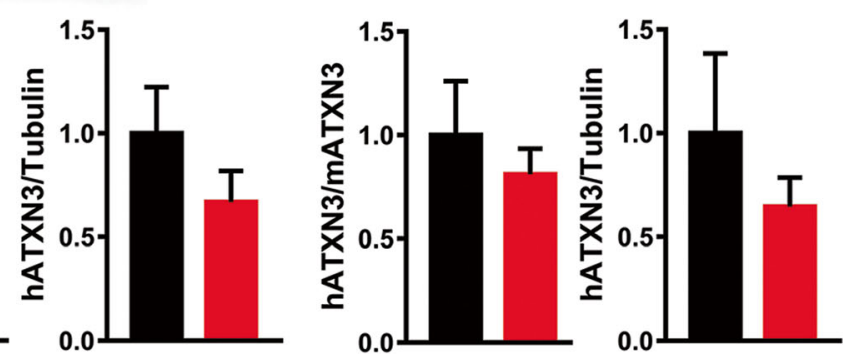

SC

WT CMVMJD135hi

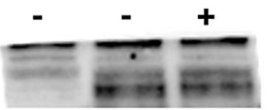

citalopram-treated CMVMJD135hi mice ( $n=4,28$ weeks). Data are presented as mean \pm SEM, normalized to WT, $P>0.05$, Student's $t$ test, Levene correction for SC data. Scale bars $=10 \mu \mathrm{m}$ (PN and LSC) and $20 \mu \mathrm{m}$ (DCN). cit citalopram, WT wild-type, PN pontine nuclei, LSC lumbar spinal cord, DCN deep cerebellar nuclei, BS brainstem, SC spinal cord, $\mathrm{CB}$ cerebellum 
There was, however, a tendency towards reduction of ATXN3 aggregation in the deep cerebellar nuclei $(\mathrm{DCN})$ of CMVMJD135hi mice $(P=0.071)$ (Fig. 4a). As seen for presymptomatic treatment of CMVMJD135 mice [20], total levels of ATXN3 protein did not change significantly upon post-symptomatic treatment of CMVMJD135hi mice in the brainstem (BS), spinal cord (SC), and cerebellum (CB) (Fig. 4b and Supplementary Fig. 5).

Post-symptomatic treatment of CMVMJD135hi mice with citalopram failed to attenuate reactive astrogliosis in the substantia nigra (SN) (Fig. 5a). Moreover, citalopram treatment exerted limited neuroprotective effects. In brain regions like the pontine nuclei $(\mathrm{PN})$ of the brainstem and in the DCN, in which at 28 weeks of age there is only a scarce neuronal loss, SSRI treatment led to a tendency of an increase in the number of NeuNpositive neurons in transgenic mice. At this age, there is also a trend towards a reduction of ChAT-positive motor neurons in the spinal cord of transgenic animals, and citalopram treatment circumvented this neuronal loss to some extent. The reduction in calbindin D-28K-positive cells seen in the Purkinje cells of the cerebellar cortex of CMVMJD135hi mice was also partially prevented by post-symptomatic citalopram treatment (Fig. 5b and Supplementary Fig. 4b). These results suggest that, unlike in early treatment, in which there was a robust suppression in mutant ATXN3 aggregation, post-symptomatic citalopram treatment promotes neuronal survival in certain regions of the mouse brain, in agreement with the observed phenotypic effects, but has a weak to absent impact on mutant protein aggregation.

\section{Discussion}

In this study, we observed that post-symptomatic treatment of MJD transgenic mice with the SSRI citalopram attenuated their loss of balance and motor dysfunction, highlighting the importance of the use of antidepressants in neurodegenerative diseases beyond the treatment of depressive symptoms.

The therapeutic capacity of citalopram in CMVMJD135hi mice that show increased symptom severity and faster progression rates compared to CMVMJD135 mice [20] further supports the advancement of this drug to clinical trials in MJD patients. As the disease progresses, however, the ability of citalopram to prevent aggregation and neuronal loss may not be sufficient to cope with the already installed neurodegenerative process; hence, an early initiation of treatment may be required. Importantly, the safety profile of SSRIs [31] allows preventive treatment of mutation carriers as well as its administration after symptom installation, with treatment efficacy being reported in two rodent models of MJD [20, 21]. Our previous results showed that presymptomatic citalopram treatment [20] exerted its effects mostly in the CNS-related symptoms rather than on the periphery (e.g., muscular strength). Here, treatment was initiated after the manifestation of core motor coordination symptoms, resulting in a percentage of therapeutic efficacy of $36 \%$, as measured at 26 weeks of age in the balance beam walk test, in contrast with the $66 \%$ obtained when treatment was initiated before the installation of motor defects. This may be due to the combination of late treatment initiation (at 11 versus 5 weeks), reduced treatment duration (17 versus 29 weeks), and increased severity of the symptoms of CMVMJD135hi mice. Both preand post-symptomatic citalopram preclinical trials resulted in preservation and/or an increase in the number of neuronal cells in affected brain regions of transgenic mice, corresponding to those affected in MJD patients but not typically assessed when studying the mechanism of action of SSRIs in major depressive disorder models [32].

While citalopram treatment presented a striking effect in protein aggregation suppression, when initiated before the appearance of ATXN3 inclusions [20], this was not so evident when the treatment was initiated post-symptomatically, at an age in which inclusions are already present. The timing of citalopram treatment initiation appears therefore to have a differential impact on ATXN3 folding/aggregation suggesting that this drug may modulate specific proteostasis subnetwork(s) acting preferentially on the prevention of formation of new aggregates and/or on the generation of new seeds, and less so by disaggregating the inclusion bodies already formed. Interestingly, and regarding the possible mechanism of action of citalopram in the context of protein aggregation diseases such as MJD, neurosensory release of 5-HT has been shown to activate cell non-autonomously the heat shock factor 1 and downstream molecular chaperones and to suppress protein misfolding in non-neuronal tissues of C. elegans [33]. On the other hand, neuronal mitochondrial proteotoxic stress induces 5-HT release from serotonergic neurons and activates the unfolded protein response of the mitochondria in C. elegans intestine cells [34]. If a similar mechanism is occurring in vertebrates, this would be consistent with an aggregation preventive rather than disruptive mechanism, which is in accordance with our data. Importantly, pre-symptomatic treatment of a second MJD transgenic mouse model (YACMJDQ84.2) [35] with citalopram also resulted in a reduction in ATXN3 inclusions and abundance, and in the modulation of certain components of cellular proteostasis, including restoration of Hsp90beta levels in brains of mice treated with this drug [21]. 

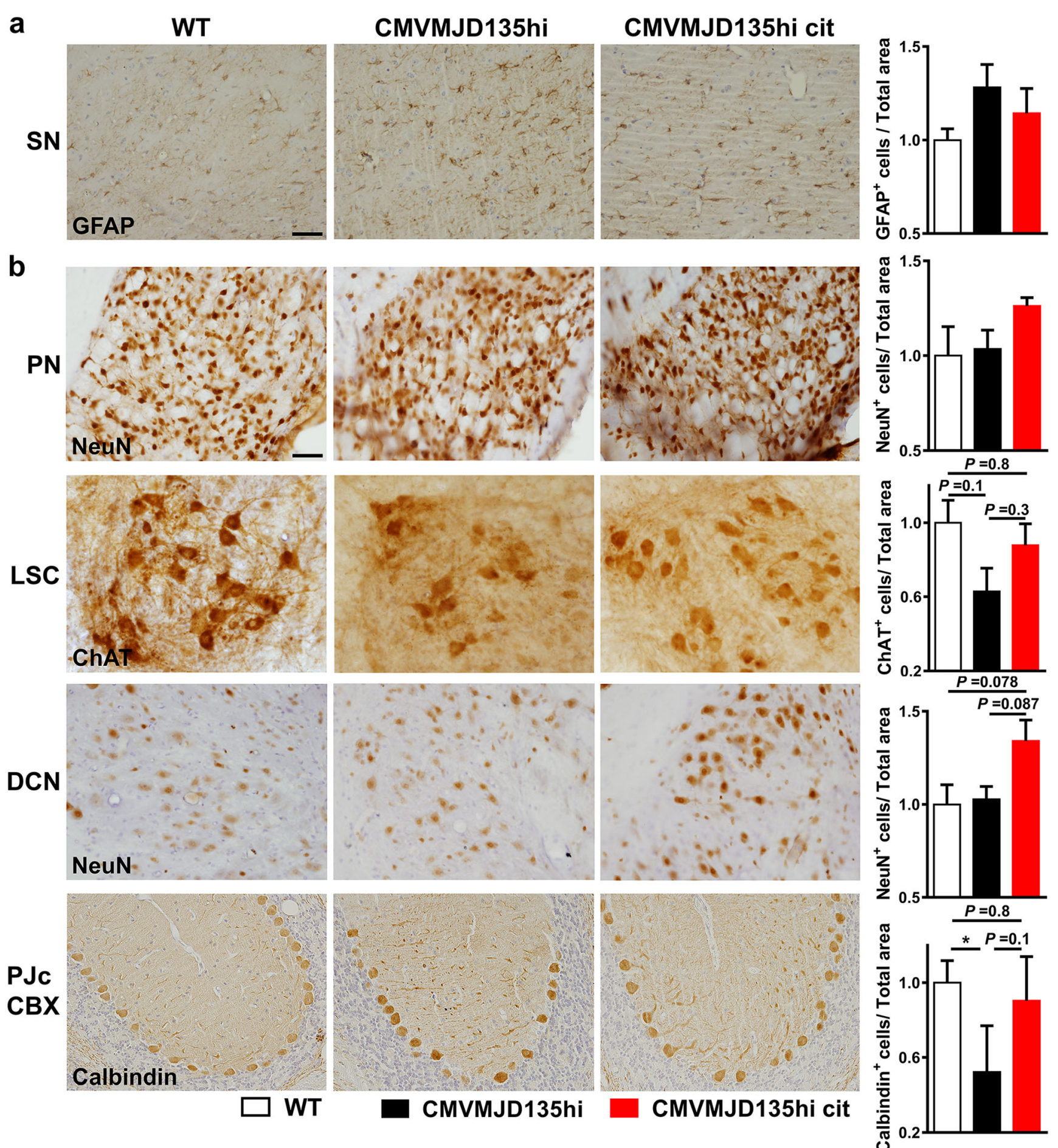

Fig. 5 Assessment of the neuroprotective effects of citalopram postsymptomatic treatment in CMVMJD135hi mice. a Immunohistochemistry and quantification of GFAP-positive cells per area in substantia nigra $(\mathrm{SN})$ from WT, vehicle- and citalopram-treated CMVMJD135hi mice ( $n=5$ per group, 28 weeks). b Immunohistochemistry and quantification of NeuN-positive cells per total area in the pontine (PN) and deep cerebellar nuclei (DCN), ChATpositive cells per total area in the lumbar spinal cord (LSC), and calbindin D-28K-positive Purkinje cells per total area in the cerebellar cortex (PJc CBX) from WT, vehicle- and citalopram-treated CMVMJD135hi mice
( $n=4$ per group, 28 weeks). At least 992 GFAP-positive cells were counted in the SN; 4973 and $3622 \mathrm{NeuN}$-positive cells were counted in the PN and DCN, respectively; 344 ChAT-positive cells were counted in the LSC, and 4052 calbindin D-28K-positive Purkinje cells were counted in the PJc CBX, per condition. Data are presented as mean $\pm \mathrm{SEM}$, normalized to WT values, $P<0.05$ (one-way ANOVA). Scale bar $=$ $50 \mu \mathrm{m}$. cit citalopram, WT wild-type, SN substantia nigra, PN pontine nuclei, LSC lumbar spinal cord, DCN deep cerebellar nuclei, PJc CBX Purkinje cells of the cerebellar cortex 
The differential impact of citalopram treatment on ATXN3 proteotoxicity in the cerebellum, brainstem, and spinal cord seen in this work and in the study by Ashraf et al. may reflect the distinct expression pattern/activation of 5-HT receptors [36-38]; therefore, it would be important to identify the specific 5-HT receptors required for citalopram's effect on toxicity and aggregation of mutant ATXN3. Previous studies in C. elegans implicated the homologs of 5HT1 and 5HT2 receptor families in this response [20]. Consistently, the antipsychotic aripiprazole was recently identified in an unbiased screen as a modulator of ATXN3 abundance. Agonism of 5HT1A and antagonism of $5 \mathrm{HT} 2 \mathrm{~A}$ receptors by aripiprazole may contribute to its therapeutic effect in Machado-Joseph disease cell and animal models [39].

Our results support a novel use for citalopram in the treatment of MJD patients. However, the therapeutic effect found after the installation of core motor symptoms in mice indicates that early initiation of treatment is preferable, bringing new insight into the design of prospective clinical trials for this and perhaps other neurodegenerative diseases.

Acknowledgments We are grateful to the members of the Maciel laboratory for sharing reagents and for critical analysis of the data and discussions on the manuscript. We thank members of the Morimoto lab for critical analysis of the results and figures. We also thank H. Lundbeck A/S for providing citalopram hydrobromide and Dr. Karina Fog for scientific discussions. We are grateful to Maria do Carmo Costa for critical review of the manuscript.

Author Contributions SE, SO, AT-C, and PM contributed to the research project conception and organization. SE, SO, SD-S, DG-C, and AT-C performed the experiments. SE, SO, SD-S, and AT-C contributed to the statistical analysis design. SE, SO, and SD-S performed the statistical analysis. SE and AT-C wrote the first draft of the article. SO, SD-S, DG-C, AT-C, and PM reviewed and criticized the manuscript. All authors read and approved the final manuscript.

Funding This work has been funded by the European Regional Development Funds (FEDER), through the Competitiveness Factors Operational Programme (COMPETE), and by National funds, through the Foundation for Science and Technology (FCT), under the scope of the project POCI-01-0145-FEDER-007038. This article has been developed under the scope of the project NORTE-01-0145-FEDER-000013, supported by the Northern Portugal Regional Operational Programme (NORTE 2020), under the Portugal 2020 Partnership Agreement, through the FEDER. This work was also supported by FCT and COMPETE through the projects [PTDC/SAU-GMG/112617/2009] (to PM) and [EXPL/BIM-MEC/0239/2012] (to AT-C), by FCT through the project [POCI-01-0145-FEDER-016818 (PTDC/NEU-NMC/3648/2014)] (to PM), by National Ataxia foundation (to PM and to AT-C), and by Ataxia UK (to PM). SE, SD-S, SO, and AT-C were supported by the FCT individual fellowships, SFRH/BD/78554/2011, SFRH/BD/78388/ 2011, PD/BD/127818/2016, and SFRH/BPD/102317/2014, respectively. FCT fellowships are co-financed by POPH, QREN, Governo da República Portuguesa, and EU/FSE.

\section{Compliance with Ethical Standards}

Conflict of Interest The authors declare that they have no conflict of interest.

\section{References}

1. Coutinho P, Andrade C (1978) Autosomal dominant system degeneration in Portuguese families of the Azores Islands. A new genetic disorder involving cerebellar, pyramidal, extrapyramidal and spinal cord motor functions. Neurology 28(7):703-709

2. Lima L, Coutinho P (1980) Clinical criteria for diagnosis of Machado-Joseph disease: report of a non-Azorena Portuguese family. Neurology 30(3):319-322

3. Kawaguchi Y, Okamoto T, Taniwaki M, Aizawa M, Inoue M, Katayama S, Kawakami H, Nakamura S et al (1994) CAG expansions in a novel gene for Machado-Joseph disease at chromosome 14q32.1. Nat Genet 8(3):221-228. https://doi.org/10.1038/ng1194-221

4. Coutinho P, Sequeiros J (1981) Clinical, genetic and pathological aspects of Machado-Joseph disease. J Genet Hum 29(3):203-209

5. Sequeiros J, Coutinho P (1993) Epidemiology and clinical aspects of Machado-Joseph disease. Adv Neurol 61:139-153

6. Rub U, Brunt ER, Deller T (2008) New insights into the pathoanatomy of spinocerebellar ataxia type 3 (Machado-Joseph disease). Curr Opin Neurol 21(2):111-116. https://doi.org/10. 1097/WCO.0b013e3282f7673d

7. Cipriani A, Purgato M, Furukawa TA, Trespidi C, Imperadore G, Signoretti A, Churchill R, Watanabe N et al (2012) Citalopram versus other anti-depressive agents for depression. Cochrane Database Syst Rev 7:CD006534. https://doi.org/10.1002/ 14651858.CD006534.pub2

8. Aboukhatwa M, Dosanjh L, Luo Y (2010) Antidepressants are a rational complementary therapy for the treatment of Alzheimer's disease. Mol Neurodegener 5:10. https://doi.org/10.1186/17501326-5-10

9. Blakely RD, Berson HE, Fremeau RT Jr, Caron MG, Peek MM, Prince HK, Bradley CC (1991) Cloning and expression of a functional serotonin transporter from rat brain. Nature 354(6348):6670. https://doi.org/10.1038/354066a0

10. Pittenger C, Duman RS (2008) Stress, depression, and neuroplasticity: a convergence of mechanisms. Neuropsychopharmacology 33(1):88-109. https://doi.org/10. 1038/sj.npp.1301574

11. Baquero M, Martin N (2015) Depressive symptoms in neurodegenerative diseases. World J Clin Cases 3(8):682-693. https://doi.org/ 10.12998/wjcc.v3.i8.682

12. Lauterbach EC, Victoroff J, Coburn KL, Shillcutt SD, Doonan SM, Mendez MF (2010) Psychopharmacological neuroprotection in neurodegenerative disease: assessing the preclinical data. J Neuropsychiatry Clin Neurosci 22(1):8-18. https://doi.org/10. 1176/appi.neuropsych.22.1.8

13. Minamiyama M, Katsuno M, Adachi H, Doi H, Kondo N, Iida M, Ishigaki S, Fujioka Y et al (2012) Naratriptan mitigates CGRP1associated motor neuron degeneration caused by an expanded polyglutamine repeat tract. Nat Med 18(10):1531-1538. https:// doi.org/10.1038/nm.2932

14. Cirrito JR, Disabato BM, Restivo JL, Verges DK, Goebel WD, Sathyan A, Hayreh D, D'Angelo G et al (2011) Serotonin signaling is associated with lower amyloid-beta levels and plaques in transgenic mice and humans. Proc Natl Acad Sci U S A 108(36): 1496814973. https://doi.org/10.1073/pnas.1107411108

15. Sheline YI, West T, Yarasheski K, Swarm R, Jasielec MS, Fisher JR, Ficker WD, Yan P et al (2014) An antidepressant decreases CSF 
Abeta production in healthy individuals and in transgenic $\mathrm{AD}$ mice. Sci Transl Med 6(236):236re234. https://doi.org/10.1126/ scitranslmed.3008169

16. Ubhi K, Inglis C, Mante M, Patrick C, Adame A, Spencer B, Rockenstein E, May V et al (2012) Fluoxetine ameliorates behavioral and neuropathological deficits in a transgenic model mouse of alpha-synucleinopathy. Exp Neurol 234(2):405-416. https://doi. org/10.1016/j.expneurol.2012.01.008

17. Duan W, Peng Q, Masuda N, Ford E, Tryggestad E, Ladenheim B, Zhao M, Cadet JL et al (2008) Sertraline slows disease progression and increases neurogenesis in N171-82Q mouse model of Huntington's disease. Neurobiol Dis 30(3):312-322. https://doi. org/10.1016/j.nbd.2008.01.015

18. Pakaski M, Bjelik A, Hugyecz M, Kasa P, Janka Z, Kalman J (2005) Imipramine and citalopram facilitate amyloid precursor protein secretion in vitro. Neurochem Int 47(3):190-195. https://doi. org/10.1016/j.neuint.2005.03.004

19. Chung YC, Kim SR, Jin BK (2010) Paroxetine prevents loss of nigrostriatal dopaminergic neurons by inhibiting brain inflammation and oxidative stress in an experimental model of Parkinson's disease. J Immunol 185(2):1230-1237. https://doi.org/10.4049/ jimmunol.1000208

20. Teixeira-Castro A, Jalles A, Esteves S, Kang S, da Silva Santos L, Silva-Fernandes A, Neto MF, Brielmann RM et al (2015) Serotonergic signalling suppresses ataxin 3 aggregation and neurotoxicity in animal models of Machado-Joseph disease. Brain 138(11):3221-3237. https://doi.org/10.1093/brain/awv262

21. Ashraf NS, Duarte-Silva S, Shaw ED, Maciel P, Paulson HL, Teixeira-Castro A, do Carmo Costa M (2018) Citalopram reduces aggregation of ATXN3 in a YAC transgenic mouse model of Machado-Joseph disease. Mol Neurobiol. https://doi.org/10.1007/ s12035-018-1331-2

22. Silva-Fernandes A, Duarte-Silva S, Neves-Carvalho A, Amorim M, Soares-Cunha C, Oliveira P, Thirstrup K, Teixeira-Castro A et al (2014) Chronic treatment with 17-DMAG improves balance and coordination in a new mouse model of Machado-Joseph disease. Neurotherapeutics 11:433-449. https://doi.org/10.1007/s13311013-0255-9

23. Nicklas W, Baneux P, Boot R, Decelle T, Deeny AA, Fumanelli M, Illgen-Wilcke B (2002) Recommendations for the health monitoring of rodent and rabbit colonies in breeding and experimental units. Lab Anim 36(1):20-42

24. Silva-Fernandes A, Costa Mdo C, Duarte-Silva S, Oliveira P, Botelho CM, Martins L, Mariz JA, Ferreira T et al (2010) Motor uncoordination and neuropathology in a transgenic mouse model of Machado-Joseph disease lacking intranuclear inclusions and ataxin-3 cleavage products. Neurobiol Dis 40(1):163-176. https:// doi.org/10.1016/j.nbd.2010.05.021

25. Carter RJ, Lione LA, Humby T, Mangiarini L, Mahal A, Bates GP, Dunnett SB, Morton AJ (1999) Characterization of progressive motor deficits in mice transgenic for the human Huntington's disease mutation. J Neurosci 19(8):3248-3257
26. Kilkenny C, Browne WJ, Cuthill IC, Emerson M, Altman DG (2010) Improving bioscience research reporting: the ARRIVE guidelines for reporting animal research. PLoS Biol 8(6): e1000412. https://doi.org/10.1371/journal.pbio.1000412

27. Rogers DC, Fisher EM, Brown SD, Peters J, Hunter AJ, Martin JE (1997) Behavioral and functional analysis of mouse phenotype: SHIRPA, a proposed protocol for comprehensive phenotype assessment. Mamm Genome 8(10):711-713

28. Rafael JA, Nitta Y, Peters J, Davies KE (2000) Testing of SHIRPA, a mouse phenotypic assessment protocol, on Dmd(mdx) and Dmd(mdx3cv) dystrophin-deficient mice. Mamm Genome 11(9): $725-728$

29. Kim HY (2013) Statistical notes for clinical researchers: assessing normal distribution (2) using skewness and kurtosis. Restor Dent Endod 38(1):52-54. https://doi.org/10.5395/rde.2013.38.1.52

30. Maciel P, Gaspar C, DeStefano AL, Silveira I, Coutinho P, Radvany J, Dawson DM, Sudarsky L et al (1995) Correlation between CAG repeat length and clinical features in Machado-Joseph disease. Am J Hum Genet 57(1):54-61

31. Nemeroff CB (2003) Overview of the safety of citalopram. Psychopharmacol Bull 37(1):96-121

32. Krishnan V, Nestler EJ (2008) The molecular neurobiology of depression. Nature 455(7215):894-902. https://doi.org/10.1038/ nature 07455

33. Tatum MC, Ooi FK, Chikka MR, Chauve L, Martinez-Velazquez LA, Steinbusch HW, Morimoto RI, Prahlad V (2015) Neuronal serotonin release triggers the heat shock response in C. elegans in the absence of temperature increase. Curr Biol 25(2):163-174. https://doi.org/10.1016/j.cub.2014.11.040

34. Berendzen KM, Durieux J, Shao LW, Tian Y, Kim HE, Wolff S, Liu Y, Dillin A (2016) Neuroendocrine coordination of mitochondrial stress signaling and proteostasis. Cell 166(6):1553-1563 e1510. https://doi.org/10.1016/j.cell.2016.08.042

35. Cemal CK, Carroll CJ, Lawrence L, Lowrie MB, Ruddle P, AlMahdawi S, King RH, Pook MA et al (2002) YAC transgenic mice carrying pathological alleles of the MJD1 locus exhibit a mild and slowly progressive cerebellar deficit. Hum Mol Genet 11(9):10751094

36. Lesch KP, Waider J (2012) Serotonin in the modulation of neural plasticity and networks: implications for neurodevelopmental disorders. Neuron 76(1):175-191. https://doi.org/10.1016/j.neuron. 2012.09.013

37. Oostland M, van Hooft JA (2013) The role of serotonin in cerebellar development. Neuroscience 248:201-212. https://doi.org/10. 1016/j.neuroscience.2013.05.029

38. Perrier JF, Cotel F (2015) Serotonergic modulation of spinal motor control. Curr Opin Neurobiol 33:1-7. https://doi.org/10.1016/j. conb.2014.12.008

39. Costa MDC, Ashraf NS, Fischer S, Yang Y, Schapka E, Joshi G, McQuade TJ, Dharia RM et al (2016) Unbiased screen identifies aripiprazole as a modulator of abundance of the polyglutamine disease protein, ataxin-3. Brain 139(11):2891-2908. https://doi. org/10.1093/brain/aww228 